\title{
MBE Growth and Magnetooptical Properties of $(\mathrm{Zn}, \mathrm{Co})$ Te Layers
}

\author{
M. Papaj, J. Kobak, J.-G. Rousset, E. Janik, A. Golnik, P. Kossacki \\ AND W. PACUSKI \\ Institute of Experimental Physics, Faculty of Physics, University of Warsaw, Hoża 69, 02-681 Warsaw, Poland

\begin{abstract}
We report on epitaxial growth of diluted magnetic semiconductor (Zn,Co)Te. Reflectivity spectra reveal excitonic transition which split under magnetic field due to giant Zeeman effect. Magnetooptical effects can be described using literature data.
\end{abstract}

PACS: 75.50.Pp, 75.30.Hx, 78.20.Ls, 71.35.Ji

\section{Introduction}

The $(\mathrm{Zn}, \mathrm{Co}) \mathrm{Te}$ is a one of diluted magnetic semiconductor (DMS) with particularly strong interaction of magnetic ions and free holes. The interaction can be described by $p-d$ exchange integral $N_{0} \beta=-3.03 \pm$ $0.15 \mathrm{eV}$ [1], so the absolute value is two or three times larger than for the other Co or Mn based DMSs (e.g. for (Cd,Mn)Te $N_{0} \beta=-0.88 \mathrm{eV}$ [2]). High optical quality bulk (Zn,Co)Te samples studied in Ref. [1] were grown using the Bridgman method, with cobalt concentration between 0.05 and $1.06 \%$. Other technique to introduce Co into ZnTe was thermal diffusion [3], which allowed infrared spectroscopy of intraionic $\mathrm{Co}^{2+}$ transitions [3, 4]. Main difficulty in doping of the ZnTe crystals during growth is high evaporation temperatures of Co as compared with the evaporation temperatures of $\mathrm{Zn}$ and Te [3]. For molecular beam epitaxy (MBE) this difficulty should be not as important as for other crystal growth techniques, but for our best knowledge, there was no reports on $\mathrm{MBE}(\mathrm{Zn}, \mathrm{Co}) \mathrm{Te}$. It would be very interesting to enhance magnetooptical effects using higher Co concentrations and epitaxial nanostructures such as quantum wells, quantum dots, or distributed Bragg reflectors. This is the reason why we equipped our new MBE machine (SVT model 26-V-3) in high temperature effusion cell.

\section{Experimental}

We present the optical characterization and molecular beam epitaxy of $(\mathrm{Zn}, \mathrm{Co}) \mathrm{Te}$ layers grown on GaAs:Si (100) substrates. The layers were grown at a constant substrate temperature $\left(360^{\circ} \mathrm{C}\right)$ with varying molecular fluxes of cobalt to provide various concentration of Co in the layers. During the growth, the samples were characterized in situ using white light reflectometry, which was used to measure the growth rate and the thickness of the layers. The smoothness of the crystal growth was monitored using reflection high-energy electron diffraction (RHEED). Each GaAs substrate was first covered with ZnTe buffer (about $100 \mathrm{~nm}$ thick). The actual (Zn,Co)Te layers had thickness of $1 \mu \mathrm{m}$ and the time of growth ranged from 1 to $1.5 \mathrm{~h}$.

It appeared that it is very difficult to grow in MBE high quality $(\mathrm{Zn}, \mathrm{Co}) \mathrm{Te}$ layer with higher Co concentration, at least much more difficult than growth of $(\mathrm{Zn}, \mathrm{Mn}) \mathrm{Te}$, which was performed just before growth of $(\mathrm{Zn}, \mathrm{Co}) \mathrm{Te}$. For $\mathrm{Zn}$ to Co flux ratio 10:1, RHEED image revealed very fast degradation of growing layer. For $\mathrm{Zn}$ to Co flux ratio 100:1, RHEED image was two-dimensional, similar to observed for typical ZnTe layers, but optical quality of $(\mathrm{Zn}, \mathrm{Co}) \mathrm{Te}$ grown in such conditions was very poor, e.g. no excitons were observed in PL or reflectivity. Finally for Zn to Co flux ratio 1000:1 not only in situ characterization but also ex situ optical study revealed good quality of the layer. For all samples we used Te-rich conditions in order to simplify the calibration of Co concentration. Growth rate was about $800 \mathrm{~nm} / \mathrm{h}$. Source of cobalt in our $\mathrm{MBE}$ is the high temperature effusion cell with typical working temperature during growth of (Zn,Co)Te 1200$1450^{\circ} \mathrm{C}$. Such a high temperature affects all other components of the MBE machine, e.g. increases substrate temperature due to radiation. Taking this into account, sputter source of cobalt can be considered as reasonable alternative for high temperature effusion cell for $\mathrm{MBE}$ growth of DMSs [5, 6].

\section{Results}

Reflectivity spectra (Fig. 1) of the most diluted (Zn,Co)Te sample revealed sharp exciton lines (FWHM $1.5 \mathrm{meV}$ ) at the low temperature of $1.7 \mathrm{~K}$. Exciton photoluminescence in the samples was not observed, probably due to nonradiative recombination through cobalt ions - effect known for wide gap DMSs [4, 7, 8]. The 
sample was examined using reflectivity at the low temperature $(T=1.7 \mathrm{~K})$ and magnetic field up to $5 \mathrm{~T}$ in the Faraday configuration (Fig. 1). We measured spectra in both $\sigma^{+}$and $\sigma^{-}$circular polarizations. Under magnetic field exciton line splits in circular polarizations due to the giant Zeeman effect $(16 \mathrm{meV})$, which is two orders of magnitude larger than normal Zeeman effect and the diamagnetic shift (both about $0.5 \mathrm{meV}$ at $5 \mathrm{~T}[9]$ ). Figure 2 shows how energy of the exciton lines depends on magnetic field. Using giant Zeeman splitting, value of $N_{0}(\alpha-\beta)=3.34 \mathrm{eV}$ and modified Brillouin function determined in Ref. [1] we determined the concentration of cobalt as equal to $0.34 \%$.

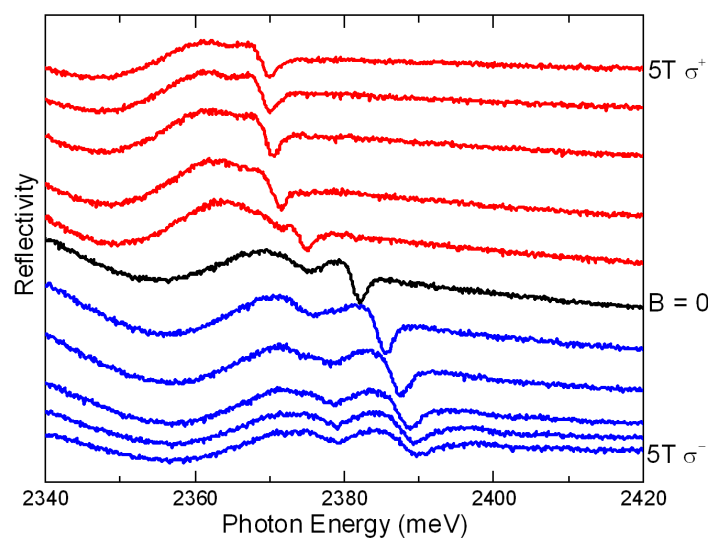

Fig. 1. Reflectivity spectra of the most diluted (Zn,Co)Te sample $\left(x_{\text {Co }}=0.34 \%\right)$ at $T=1.7 \mathrm{~K}$. Black line in the middle corresponds to no external magnetic field, red (upper) and blue (lower) lines correspond to magnetic field between $1 \mathrm{~T}$ and $5 \mathrm{~T}$ in steps of $1 \mathrm{~T}$ for $\sigma^{+}$and $\sigma^{-}$polarizations, respectively. Exciton structure shifts due to the giant Zeeman effect.

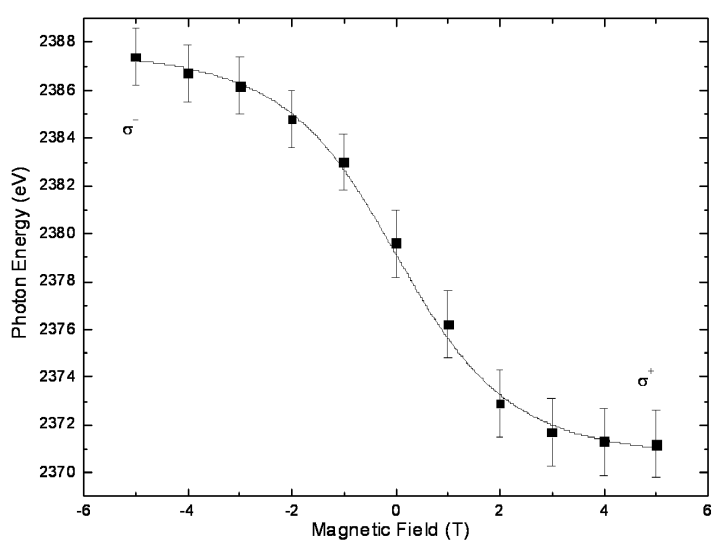

Fig. 2. Excitonic transition energy vs. magnetic field determined from spectra shown in Fig. 1. The solid line is a fit with a modified Brillouin function described in Ref. [1], it corresponds to cobalt concentration $x_{\mathrm{Co}}=$ $0.34 \%$.
We conclude that $(\mathrm{Zn}, \mathrm{Co}) \mathrm{Te}$ layers can be grown in MBE equipped with high temperature effusion cell. It was shown for diluted samples $\left(x_{\mathrm{Co}}=0.34 \%\right)$, growth of good optical quality layers with higher concentrations will be much more challenging. However even low concentration $x_{\text {Co }}=0.34 \%$ can be useful for magnetooptical experiments because giant Zeeman effect for such a diluted sample results in significant exciton splitting, $16 \mathrm{meV}$ at saturation.

\section{Acknowledgments}

This work was supported by Polish public funds in years 2011-2017 (NCN projects DEC-2011/01/B/ST3/ 02406 and DEC-2011/02/A/ST3/00131, and NCBiR project LIDER/30/13/L-2/10/NCBiR/2011). Project was carried out with the use of CePT, CeZaMat and NLTK infrastructures financed by the European Union - the European Regional Development Fund within the Operational Programme "Innovative economy" for 20072013.

\section{References}

[1] M. Zielinski, C. Rigaux, A. Lemaître, A. Mycielski, J. Deportes, Phys. Rev. B 53, 674 (1996).

[2] J.A. Gaj, R. Planel, G. Fishman, Solid State Commun. 29, 435 (1979).

[3] Yu.F. Vaksman, Yu.A. Nitsuk, V.V. Pavlov, Yu.N. Purtov, A.S. Nasibov, P.V. Shapkin, Semicond. Electron. Opt. Prop. Semicond. 41, 660 (2007).

[4] W. Pacuski, D. Ferrand, J. Cibert, C. Deparis, J.A. Gaj, P. Kossacki, C. Morhain, Phys. Rev. B 73, 035214 (2006).

[5] X. Liu, A. Petrou, B.T. Jonker, J.J. Krebs, G.A. Prinz, J. Warnock, J. Appl. Phys. 67, 4796 (1990).

[6] B.T. Jonker, G.A. Prinz, J. Appl. Phys. 69, 5676 (1991).

[7] W. Pacuski, J. Suffczynski, P. Osewski, P. Kossacki, A. Golnik, J.A. Gaj, C. Deparis, C. Morhain, E. Chikoidze, Y. Dumont, D. Ferrand, J. Cibert, T. Dietl, Phys. Rev. B 84, 035214 (2011).

[8] W. Pacuski, "Optical Spectroscopy of Wide-Gap Diluted Magnetic Semiconductors", chapter in Introduction to the Physics of Diluted Magnetic Semiconductors, Ed. J.A. Gaj, J. Kossut, Springer Series in Materials Science, Vol. 144, Springer, Heidelberg 2010, p. 37.

[9] K. Gietka, J. Kobak, J.-G. Rousset, E. Janik, T. Słupiński, P. Kossacki, A. Golnik, W. Pacuski, Acta Phys. Pol. A 122, 1056 (2012). 